\title{
CONTROL ALGORÍTMICO: EN ORGANIZACIONES Y SOCIEDAD
}

\section{CONTROL: IN ORGANIZATIONS AND SOCIETY}

\author{
Óscar Emilio Mora Navarro ${ }^{1}$
}

\begin{abstract}
Palabras clave:
Control, control algorítmico, control racional, control administrativo, prácticas administrativas.
\end{abstract}

\begin{abstract}
Resumen
Ante un ambiente de cambio permanente, la transformación digital ha impulsado la implementación de tecnologías en las organizaciones y la sociedad, para maximizar el valor del trabajo, y está contribuyendo con nuevas prácticas de control. Con visión positiva, los empresarios están empleando el control algorítmico para mejorar la gestión porque facilita el control, en comparación con los instrumentos manuales o semiautomatizado usados en el siglo XX. Por su parte, la digitalización y la inteligencia artificial, entre otras, generan preocupación sobre el futuro del trabajo, producen inseguridad (inseguridad algorítmica) y las personas se están resistiendo individual y colectivamente al control algorítmico (algoactivismo). Lo anterior, lleva a plantearse, por ejemplo, cómo los algoritmos están redefiniendo el control organizacional, cómo influyen en el clima organizacional y en el bienestar emocional de los colaboradores o cómo los algoritmos pueden considerar la cultura $\mathrm{y}$ los factores ambientales al ejercer el control.
\end{abstract}

Códigos JEL: M10

\begin{abstract}
In an environment of permanent change, digital transformation has promoted the implementation of technologies in organizations and society, to maximize the value of work, and is contributing with new control practices. With a positive view on the part of employers, algorithmic control is being used to improve management because it facilitates control, compared to manual or semi-automated instruments used in the 20th century. Digitization and artificial intelligence, among others, cause concerns about the future of work, produce insecurity (algorithmic insecurity) and people are individually and collectively resisting algorithmic control (algoactivism). Therefore, we must consider, for example, how algorithms are redefining organizational control, how it influences the organizational climate and the emotional well-being of employees, or how algorithms can consider the culture and environmental factors when exercising control.
\end{abstract}

\footnotetext{
${ }^{1}$ Ingeniero de Sistemas y Magíster en Administración Universidad Nacional de Colombia. Carrera 52 22-39 apartamento 1203 Torre A Scala 25. Bogotá D.C. Colombia.

Email: omoranav@hotmail.com
} 



\section{INTRODUCCIÓN}

La transformación digital ha impulsado la implementación de tecnologías y está modificando la forma en que operan las empresas y los mercados. Así mismo, contribuyendo con nuevas prácticas de control, en la cuales se empieza a entrever las deficiencias porque no existe un modelo organizacional correcto, sino que cada uno tiene ventajas, limitaciones y aplicaciones específicas.

La mayor parte de la investigación en administración y economía enfatiza en los beneficios de usar algoritmos para mejorar la asignación y la coordinación, la toma de decisiones y el aprendizaje organizacional. Estos análisis se centran sobre el impacto de los algoritmos en términos de valor económico derivado de mayor eficiencia, ingresos, e innovación. Aunque, se está estudiando el impacto de los algoritmos en la relación empleador-trabajador (Kellogg, et al., 2020, p. 366) y cómo genera una forma novedosa de inseguridad, denominada "Inseguridad algorítmica" (Wood y Lehdonvirta, 2021, pp. 12).

El control algorítmico es el nuevo terreno del control, en el cual los trabajadores se resisten, para defender su autonomía frente a un control más estricto por parte del empleador, utilizando un conjunto de tácticas emergentes llamadas algoactivismo (Kellogg, et al., 2020, p. 368). Dado que las tecnologías tienen el potencial de transformar el control organizacional, con implicaciones significativas para los colaboradores, es imperioso explorar la intersección entre tecnologías emergentes, la naturaleza cambiante del trabajo y el control y las implicaciones para la organización (Leonardi y Vaast, 2017; Kellogg, et al., 2020, p. 367).

En este sentido, los objetivos de este artículo son revisar cómo el control ha evolucionado con la incorporación de la tecnología o cómo los empresarios están utilizando algoritmos para el control organizacional y plantear los debates que generan la medición, la vigilancia y el control algorítmico.

\section{Control en Administración}

Cada organización debe alcanzar sus objetivos apalancada en sus competencias, recursos y con eficiencia, economía, competitividad y sostenibilidad. Es el administrador el responsable de obtener los resultados por la cooperación efectiva de sus colaboradores, a través de la administración (Chiavenato, 2019), la cual es el proceso de: Planear, organizar, dirigir y controlar. En planear se define misión, visión, objetivos y estrategias; en organizar la estructura organizacional, los procedimientos, se asignan recursos, etc.; en dirigir se ejecuta lo planeado para obtener los resultados previstos, y en controlar se evalúa y realizan los ajustes (Bernal Torres, 2014, p. 169).

En general, el control consiste en evaluar y corregir el desempeño de las actividades, de conformidad con el plan previsto y garantizar que los recursos se usen de manera eficaz y eficiente para alcanzar los objetivos empresariales (Stoner, 1996, pp. 610-611). En igual sentido, en Koontz (2012), se define como la "medición y corrección del desempeño para garantizar que los objetivos de la empresa y los planes diseñados para alcanzarlos se logren".

\section{Prerrequisitos de los sistemas de control}

Los controles deben basarse en planes claros, completos e integrados que se convierten en estándares para medir las acciones deseadas. Resulta inútil intentar diseñar controles sin tomar en consideración dichos planes y la forma cómo se elaboran (Koontz, et al., 1986, p. 597).

Dado que el propósito del control es medir las actividades y emprender las acciones para asegurar el logro de los planes, se debe saber en qué parte recae la responsabilidad por las desviaciones y la iniciación de acciones correctivas (Koontz, et al., 1986, pp. 610-611).

\section{Proceso básico de control}

Las tres etapas del control son: Establecer estándares, medir el desempeño en comparación con esos estándares y corregir las desviaciones respecto a estándares y planes. Los estándares son 
criterios de desempeño y son de muchas clases, por ejemplo: metas u objetivos verificables, en términos cuantitativos o cualitativos. Estos estándares de metas se plantean en términos físicos, tales como cantidades de productos, unidades de servicio, horas de trabajo, velocidad o volumen de rechazos (Koontz, et al., 2012, pp. 496-498).

La segunda etapa, medir o evaluar el desempeño en comparación con los estándares, permite identificar las desviaciones para emprender las acciones apropiadas. Si los estándares se establecen en forma adecuada y se determina con exactitud qué se hace, resulta sencilla la evaluación del desempeño real o esperado (Koontz, et al., 2012, p. 496).

El proceso se completa cuando se emprenden acciones correctivas. Con frecuencia, se piensa que el control detecta y corrige desviaciones negativas o un desempeño deficiente. Aunque, en ocasiones las desviaciones pueden ser positivas y, en este caso, es necesario revisar los estándares, para determinar si el resultado es una inexactitud o un desempeño superior (Koontz, et al., 2012, pp. 496-497).

\section{Control reactivo o proactivo}

Cuando aumenta la complejidad de los sistemas se considera las interrelaciones de muchas variables, la gerencia debe concentrarse en los resultados y el desempeño organizacional y el control se emplea para un incremento de productividad y un mejor rendimiento.

En el esfuerzo de implantar las normas de desempeño, que mediante la retroalimentación informativa comparen el desempeño efectivo con los planes, se puede caminar hacia el futuro mirando el pasado (control reactivo). Otra alternativa es especificar a dónde se quiere llegar y tratar de lograrlo (control preventivo o proactivo), diseñando un futuro deseable y la manera de avanzar hacia él con efectividad (Koontz, et al., 2012, p. 505).

Independiente de la alternativa, los controles reflejan el patrón de Organización y el Administrador debe interesarse por lo que afecte el desempeño y los resultados de la Organización, las operaciones que requieren control y las alternativas para lograr la meta.

\section{Información y control en tiempo real}

El propósito de la información en la Organización no es sólo proporcionar conocimiento, sino dar pautas para tomar la acción acertada. Por esto, se deben eliminar los datos innecesarios $\mathrm{y}$ disponer, analizar e interpretar los que resulten útiles. Quizás sea necesario presentarlos de diferentes maneras para distintos propósitos y enfocarlos hacia los cargos ejecutivos, para la toma de decisiones.

En la actualidad, la calidad de la decisión no depende de la oferta de información, sino de la demanda de ésta. El problema principal no es la libre disposición de datos, sino el modelo o esquema que los buscó. Los administradores requieren, para lograr un control administrativo, un sistema que indique en forma oportuna las desviaciones, porque los sucesos imprevistos pueden ocurrir y alterar los resultados deseados. Por esta razón, la administración exige apoyarse en información y control en tiempo real, para lograr una innovación continua.

Uno de los avances interesantes, obtenidos con el uso de las tecnologías, es la de obtener información en tiempo real, es decir, información de lo que sucede mientras está ocurriendo (Koontz, et al., 2012, pp. 502-503). En estas condiciones, el valor del trabajo reside en el contenido creador o transformador, el cual se mide por el valor de su resultado. A una Organización, que aspira a una dinámica de progreso continuo, lo peor que le puede suceder es aportar horas de personal sin iniciativa $y$ creación.

En el Misántropo de Moliére, Oronte desea la opinión de Alceste sobre un soneto, pero para conciliar la indulgencia de su juez menciona el poco tiempo empleado en escribirlo. A esto, Alceste replica: "el tiempo nada tiene que ver en el asunto" (Moliere, 1997, pp. 33-34). 


\section{Acciones correctivas o cambio}

Una acción correctiva indica que algo no anda bien y se requiere volver al funcionamiento normal. El cambio implica la elección de una acción en el cual el futuro es distinto del pasado. A diferencia de la acción correctiva, existe una preferencia por el desvío, la novedad y el abandono de las pautas anteriores (Foerster, 1996, pp. 174 - 175). ¿Qué es más eficaz, para ganar en un juego, que cambiar las reglas de acuerdo con la conveniencia?

La competitividad está en manos de las organizaciones que mantienen una dinámica de progreso, cambiando la norma de eficiencia. El estándar es un punto de paso, en lugar de una referencia durable.

\section{El terreno histórico del control}

El control organizacional se ha desarrollado entre dos corrientes: El control normativo, en el cual los administradores pueden regular a los colaboradores atendiendo su comportamiento, sus pensamientos y emociones, para conseguir productividad y eficiencia. Al ganar los corazones y las mentes, los administradores logran la autoridad moral, una de las formas más sutiles de control. En este ámbito normativo estarían: Mejoramiento Industrial, capitalismo de Bienestar/Relaciones Humanas y cultura organizacional (Barley y Kunda, 1995, p. 81).

La segunda, el control racional, "centra su atención en el control del comportamiento de los individuos, considerados como partes constitutivas del proceso productivo del cual participan. El interés de las retóricas inscritas en esta corriente se concentra entonces en la regulación de los procesos productivos" (Garcés Uribe, 2002, p. 26). En este ámbito racional estaría: La Administración Científica y el Racionalismo de Sistemas (Barley y Kunda, 1995, p. 81).

La gerencia y los miembros de cada Organización, cuando lo consideren pertinente, deben identificar, analizar, discutir e implementar nuevos modelos administrativos y de control de gestión. En este caso, el control algorítmico es una nueva forma de control racional (Kellogg, et al., 2020, p. 369).

\section{Algoritmos: Valor Económico}

Aunque el término algoritmo se popularizó con la era informática, proviene de Mohammed alKhoWârizmi, matemático persa que vivió durante el siglo IX. La palabra algorismus corresponde a la traducción del apellido al latín, la cual derivó en algoritmo, que puede definirse como un "método para resolver un problema" (Joyanes Aguilar, 2020, p. 59).

El lenguaje de programación y las computadoras permiten ejecutar el algoritmo y efectuar el proceso correspondiente. En la ciencia de la computación, el diseño de algoritmos es un aspecto primordial para tener en cuenta (Joyanes Aguilar, 2020, p. 60). Los algoritmos se están empleando para reemplazar los procesos en los cuales hay intervención humana porque son más baratos, fáciles de mantener y controlar (Del Castillo, 2018).

Por lo anterior, las investigaciones se han enfocado en lo económico y operativo, el valor de los algoritmos para las organizaciones. En particular, se ha enfatizado en cómo los empleadores pueden utilizar algoritmos para facilitar la toma de decisiones (algoritmos para optimizar los objetivos económicos $\mathrm{y}$ organizativos), coordinación y aprendizaje organizacional (Kellogg, et al., 2020, p. 368).

Los estudios muestran cómo las tecnologías permiten a las personas tomar decisiones más precisas que antes porque las organizaciones están recopilando datos más detallados sobre cómo los clientes interactúan con los productos (Glynn y Prabhakar, 2018; Kellogg, et al., 2020, p. 368) o los algoritmos de aprendizaje automático pueden predecir preferencias (Boyle y Beck, 2018; Kellogg, et al., 2020, p. 368). En algunos casos, los análisis automatizados no consideran a los humanos en el proceso de toma de decisiones.

Cuando los empresarios recopilan datos de manera automática, por ejemplo, con el internet de las cosas, los algoritmos pueden ofrecer 
información o ideas personalizadas para diferentes individuos, equipos y proyectos. $\mathrm{Si}$ bien se debe considerar el riesgo de que la tecnología inteligente reemplace las capacidades humanas, es importante darse cuenta de que también tiene un enorme potencial para aumentarlas: puede impulsar la creatividad en el trabajo, ayudar a ser mejores estudiantes, brindar atención médica de manera eficaz, hacer que las sociedades sean más sostenibles e informar mejor para la toma de decisiones (Landay, 2019; Kellogg, et al., 2020, p. 369).

\section{Control Algorítmico: El nuevo Terreno del Control en Disputa}

Los algoritmos utilizados generan un sistema de puntuación (cuando se obtiene una puntuación elevada existe una opción, pero si no se consigue se deniega). Por ejemplo, en la obtención de un puesto de trabajo, la admisión en la universidad o la obtención de una tarjeta de crédito. De manera general, se asigna una puntuación a la cual no se tiene acceso. Esto no solo es algo injusto para el individuo, sino que este sistema de decisión puede ser nocivo también para la sociedad (Del Castillo, 2018).

Con los algoritmos se está buscando trascender el prejuicio humano con una herramienta más precisa, pero se puede aumentar la desigualdad de manera progresiva, con efectos negativos en la sociedad. Quien tiene el control despliega el algoritmo, por ejemplo, en China, los sistemas de puntuaciones sociales son intentos explícitos de controlar a los ciudadanos. Se está ante una sociedad en la cual el Gobierno utiliza los algoritmos para ejercer control y vigilancia (Del Castillo, 2018).

Así mismo, en el ámbito organizacional, se están utilizando algoritmos para controlar a los trabajadores a través de seis mecanismos llamados las "6 R" (restricción, recomendación, registro, rating, reemplazo y recompensa): los empresarios pueden usar los algoritmos para dirigir a los colaboradores a través de restricciones y recomendaciones, evaluarlos mediante el registro y el rating (clasificación), y disciplinarlos con reemplazo y recompensas (Kellogg, et al., 2020, p. 372).
Los empresarios están empleando el control algorítmico para especificar qué debe realizarse, en qué orden y período de tiempo y con cuál grado de precisión. Al igual que en anteriores formas de control racional, los empleadores pueden utilizar la tecnología para priorizar decisiones específicas para que los trabajadores las implementen (Kellogg, et al., 2020. p. 372). Por ejemplo, las plataformas digitales, como Uber o Amazon Mechanical Turk, utilizan algoritmos para gestionar a los trabajadores autónomos (Jiang, et al., 2021).

En Uber, a pesar de la promesa de "Tú decides cuándo y qué tan seguido conducir" o "Elige cómo y cuándo quieres recibir tus ganancias" (Uber Technologies, Inc., 2021), un análisis de las experiencias de los conductores revela el poco control que tienen sobre los aspectos críticos de su trabajo y cuánto control tiene Uber sobre su labor (Rosenblat y Stark, 2016, p. 3762).

Aunque la esperanza es que los algoritmos mejoren la precisión y objetividad de las decisiones, estos pueden afectar las condiciones y el bienestar de los colaboradores, quienes se sienten frustrados cuando las recomendaciones algorítmicas no son claras para ellos (Kellogg, et al., 2020, p. 374). Por ejemplo, cuando el sistema de Uber no permite ver a los conductores el destino de un pasajero, antes de aceptar el viaje, esto dificulta determinar qué tan rentable será el servicio. La aceptación ciega de pasajeros es parte del diseño del algoritmo porque el sistema niega a los conductores el acceso a cierta información clave que ayudaría a tomar decisiones informadas (Rosenblat y Stark, 2016, p. 3771).

En general, las aplicaciones controlan ubicación, desplazamientos y tiempos de arribo, entrega y espera de los trabajadores autónomos; realizan evaluación continua y estricta de su desempeño con una puntuación que se actualiza con cada reparto o viaje, y la valoración recibida condiciona la posibilidad de elegir horarios en el futuro. El mecanismo es de caja negra: los colaboradores desconocen cómo se afecta su valoración con las ausencias, el no trabajar en horarios de alta demanda o aumentar el tiempo de 
reparto o viaje. Aunque sí saben y experimentan las consecuencias de dichas conductas (Morales Muñoz y Abal Medina, 2020, p. 5). El control algorítmico permite a los jefes rastrear lo que los empleados están haciendo, pero limitan la comprensión de los colaboradores sobre la estrategia empresarial (Kellogg, et al., 2020, p. 383).

El exceso de gestión algorítmica se siente en las personas como apatía, desconfianza, violencia, desconexión, impotencia, entre otras. Las personas experimentan vulnerabilidad $\mathrm{y}$ miedo como resultado de trabajar en un entorno inestable, en el cual las plataformas utilizan puntuaciones generadas por los clientes para calificarlos y algoritmos para aplicar las consecuencias de esas calificaciones (Wood y Lehdonvirta, 2021, pp. 3-4). Esto crea una "crisis participativa" porque el individuo carece de canales adecuados de comunicación o bucle de retroalimentación.

Así mismo, cuando las personas están dirigidas por un algoritmo, que perciben como injusto, aumenta su disposición a participar en comportamiento no éticos. En las plataformas digitales, los trabajadores con bajo rendimiento se excluyen de las actividades de la plataforma y se sustituyen por otros. Aunque el reemplazo es un medio eficaz para asegurar la producción, provoca resistencia individual y colectiva al control algorítmico, la cual se ha denominado algoactivismo (Kellogg, et al., 2020, p. 366; Jiang, et al., 2021).

Al ver los algoritmos como instrumentos de control, se perciben no sólo como herramientas que facilitan la eficiencia y mejoran los intercambios de información, sino como instrumentos controvertidos que pueden incluir preferencias ideológicas, implementadas de acuerdo con intereses particulares poderosos, o tener sesgos de entrenamiento que conducen a modelos discriminatorios. En la gestión algorítmica, los empresarios tienen la ilusión de un control gerencial, respaldada por una supervisión detallada al utilizar un método omnipresente y automático de supervisión y disciplina de los colaboradores, que es un método de control costo-efectivo (Woodcock, 2020, p.
69), pero lejos de ser satisfactorio para todas las partes.

Las condiciones de trabajo, en la mayoría de los casos precarias, producidas por las nuevas tecnologías digitales, contribuyen una forma novedosa de inseguridad denominada "Inseguridad algorítmica" (Wood y Lehdonvirta, 2021, pp. 1-2). Si bien las plataformas pueden ofrecer altos niveles de flexibilidad, autonomía y variedad de tareas, estos beneficios a menudo se combinan con salarios bajos, aislamiento social, horarios laborales irregulares, y agotamiento (Perelman, 2020).

Aunque los estudios se han enfocado en la eficiencia y el logro de los objetivos organizacionales, se está abordando cómo el uso de algoritmos puede afectar de forma negativa a los trabajadores o cuál es la responsabilidad empresarial, sobre todo cuando los administradores mencionan que los sistemas de control algorítmico responden a una automatización a la cual no tienen acceso, porque pueden estar minimizando de manera deliberada su papel en la calibración e intervención en la arquitectura de los sistemas (Kellogg, et al., 2020, p. 383).

Es necesario que, desde la administración, las ciencias de la información, la sociología, las ciencias de la comunicación, la psicología, las ciencias jurídicas, entre otras, se siga analizando las implicaciones de los algoritmos en términos de vigilancia y discriminación, teniendo en cuenta que se está más presionado para maximizar la exposición y la aprobación. Se debe ir más allá de la obsesión de la economía algorítmica con las "métricas", "compromiso" e "impacto", porque existe un delicado equilibrio entre el uso de nuevas tecnologías y ser utilizado por ellas (Pasquale, 2015; Kellogg, et al., 2020, p. 367).

En la actualidad, algunos dispositivos reconocen la voz, pero su comprensión se limita a enunciados aislados que desencadenan tareas predefinidas. Sin embargo, en el futuro la tecnología estará en capacidad de realizar un mayor control cuando pueda reconocer la conversación humana (continua, contextual y multimodal: se habla en voz alta, se hacen gestos 
con las manos y referencia al entorno, e incluso se toca, hace clic y escribe en paralelo) (Landay, 2019). Ante este panorama, ¿Qué destrezas deben desarrollar las personas para lograr una ventaja competitiva?

Existen tres áreas sobre las cuales se lleva la delantera: la "ideación", la capacidad de discutir ideas, ser creativos o tener un sentido del propósito; la "comunicación compleja", la capacidad de hablar o escribir, escuchar o leer, de forma sofisticada, y el "reconocimiento de patrones amplios", la facultad de procesar gran información multisensorial y responder de forma adecuada. Creatividad, comunicación compleja y pensamiento crítico son destrezas que se deben desarrollar desde las aulas (Beard, 2019, p. 57).

\section{Relaciones laborales}

En comercialización y distribución, las plataformas convocan a personas como "socios" o "colaboradores" y promocionan autonomía (la persona realiza la actividad cuando desee), cuando en realidad están sometiendo al trabajador autónomo a parámetros de rendimiento $\mathrm{y}$ de calificaciones que los fuerzan a estar disponible (Perelman, 2020).

En estos casos, se reemplaza el control laboral directo por un sistema de control algorítmico que, mediante rankings o puntajes, condiciona los pedidos recibidos y las tarifas cobradas; conlleva largas jornadas, durante la semana; no se garantiza una retribución mínima; ante una incapacidad se dejan de percibir ingresos, y se tiene escasa cobertura frente accidentes o riesgos laborales (Perelman, 2020).

Por tal razón, es el ámbito de las relaciones laborales el primero en reaccionar ante un riesgo evidente, el del control privado de los algoritmos que puede generar concentración de poder $\mathrm{y}$ discriminación. De manera específica, España es pionera, al reconocer el derecho a recibir información sobre parámetros, reglas e instrucciones de los algoritmos o inteligencia artificial empleados para tomar decisiones que afectan las condiciones de trabajo (Coscubiela, 2021, párr. 12).
Se trata de una novedad importante y pionera que abre la puerta a la disputa por la transparencia y el control de los algoritmos. Sin embargo, los derechos reconocidos en las leyes se aplican, defienden y ensanchan en la medida en que se ejercen. Por tanto, es necesario el control social de los algoritmos, el cual no puede considerarse un bien privado porque afecta derechos fundamentales de las personas. Por supuesto, esta es una batalla que es necesario dar de manera global para avanzar en la regulación de los algoritmos (Coscubiela, 2021).

\section{CONCLUSIONES}

La transformación digital exige a las organizaciones innovar en las operaciones, en los procesos de producción y en los mecanismos de control. Cuando se logra entender a la Organización y el objetivo del control, se puede ser capaz de encontrar mecanismo de control que permitan una acción orientada, precisa y congruente.

El control ha evolucionado con la incorporación de la tecnología. Cada vez más, los empresarios están utilizando algoritmos para el control organizacional y las deficiencias en los mecanismos de control emergen, como una dimensión posible, con efectos en los trabajadores $\mathrm{y}$ en la sociedad. No existe un modelo organizacional correcto, sino que cada uno tiene ventajas, limitaciones y aplicaciones específicas. Cualquier criterio u ordenamiento es una cuestión de elección.

La gerencia y los miembros de cada Organización, cuando lo consideren pertinente, deben identificar, analizar, discutir e implementar nuevos modelos administrativos y de control de gestión. El control algorítmico puede ser más completo, instantáneo, interactivo y desconocido por parte de los colaboradores que otras formas de control racional y puede permitir mayor desintermediación de administradores.

El exceso de gestión algorítmica se siente en las personas como apatía, desconfianza, violencia, desconexión, impotencia, entre otras. Esto crea una "crisis participativa" porque se excluye al individuo de la participación en el 
proceso debido a la ausencia de adecuados canales de comunicación o bucle de retroalimentación. En este contexto, el empleado no puede desarrollar ningún tipo de creatividad porque todo lo que debe hacer le es ordenado.

El control algorítmico puede eliminar a la supervisión humana en el ámbito laboral y los trabajadores se están resistiendo de manera individual o colectiva en torno a la equidad algorítmica, la responsabilidad, la transparencia, la pérdida de privacidad, la discriminación y la propiedad de los datos.

Los efectos de las intervenciones de los Administradores en una Organización deben considerarse desde la perspectiva de la moral, para conocer si los intereses a los cuales se está sirviendo son admisibles o reprobables, si corresponden a los valores reconocidos o los contradice.

La legislación debe garantizar el derecho a recibir información sobre los parámetros, reglas e instrucciones en la que se basan los algoritmos utilizados por las empresas para adoptar decisiones que afectan a las personas y pueden acentuar las desigualdades. Se tiene un sistema que se diseñó para la revolución industrial y se debe aprender a desarrollar el potencial humano utilizando la inteligencia artificial y la fusión de otras tecnologías.

Es necesario que, desde la administración, las ciencias de la información, la sociología, las ciencias de la comunicación, la psicología, las ciencias jurídicas, entre otras, se siga analizando las implicaciones del control algorítmico; qué pueden hacer empresarios y trabajadores para mitigar los resultados negativos en las personas que son sometidos a la dirección, la evaluación y la disciplina algorítmica; cómo los algoritmos están redefiniendo el control organizacional; cómo influye en el clima organizacional y en el bienestar emocional de las personas, y cómo los algoritmos pueden considerar la cultura y los factores ambientales.

Finalmente, aunque se mantiene la confianza en los algoritmos, se puede admitir que son imperfectos e incluyen sesgos de entrenamiento, en algunos casos, por ejemplo, racistas o sexistas. No se puede abandonar la transformación digital, la automatización y el uso de inteligencia artificial, pero sí prepararnos para el cambio y exigir rendición de cuentas.

\section{REFERENCIAS}

Barley, S. y Kunda G. (1995). Plan y dedicación: Oleadas de las Ideologías del control normativo y racional en el discurso administrativo. Innovar, 6 , 80-102.

Beard, A. (2019). Otras formas de aprender. Qué funciona en educación y por qué. Barcelona: Plataforma Editorial.

Bernal Torres, C. A. (2014). Introducción a la administración de las Organizaciones: enfoque global e integral. (2a. ed.). Bogotá: Pearson Educación.

http://ezproxy.biblored.gov.co:2057/?il=3406

Boyle, E. y Beck J. (2018, 28 de junio). Understanding latent style. Multithreaded. Stitch Fix.

https://multithreaded.stitchfix.com/blog/2018/06/ 28/latent-style/

Chiavenato, I. (2019). Introducción a la teoría general de la administración. (10a. ed.). México: McGraw-Hill.

http://ezproxy.biblored.gov.co:2057/?il=9167

Coscubiela, J. (2021, 12 de mayo). La disputa por el control del algoritmo. El diario.es. https://www.eldiario.es/opinion/zonacritica/disputa-controlalgoritmo_129_7927909.html

Del Castillo, C. (2018, 30 de octubre). "La próxima revolución política será por el control de los algoritmos". World Economic Forum, https://es.weforum.org/agenda/2018/10/laproxima-revolucion-politica-sera-por-el-controlde-los-algoritmos

Etkin, J. (1996). La empresa competitiva: grandeza y decadencia, el cambio hacia una organización vivible. Santiago: Mc Graw-Hill. 
Foerster, H. (1996). Las semillas de la Cibernética: Obras escogidas. Edición de Marcelo Pakman y presentación de Carlos Sluzki. Barcelona: Gedisa.

Garcés Uribe, O. L. (2002). La ideología de control en el pensamiento administrativo. ADMinister, 1, 24-38. https://publicaciones.eafit.edu.co/index.php/admi nister/article/view/667

Glynn, P. y Prabhakar, D. (2018, 08 de noviembre). Your client engagement program isn't doing what you think it is. MultiThreaded. Stitch

Fix. https://multithreaded.stitchfix.com/blog/2018/11/ 08/bandits/

Grigera, J. y Nava, A (2021). El futuro del trabajo en América Latina: crisis, cambio tecnológico y control. El Trimestre Económico, 88(4), 10111042.

https://www.proquest.com/docview/2580355684 /fulltext/3ED6DC2E22B84F26PQ/1?accountid= 31491

Jiang, J. Adam, M., y Benlian, A. (2021). Algoactivistic Practices in Ridesharing - A Topic Modeling \& Grounded Theory Approach. ECIS Research Papers.

1.

https://aisel.aisnet.org/ecis2021_rp/1/

Joyanes Aguilar, L. (2020). Fundamentos de programación: algoritmos, estructura de datos y objetos. (5a. ed.). México: McGraw-Hill. http://ezproxy.biblored.gov.co:2057/?il=10409

Kellogg, K., Valentine, M. y Christin, A. (2020). Algorithms at work: The new contested terrain of control. Academy of Management Annals, 14(1), 366-410. http://www.angelechristin.com/wpcontent/uploads/2020/01/Algorithms-atWork_Annals.pdf

Koontz, H., Weihrich, H. y Cannice, M. (1986). Administración (8a. ed.), México: McGraw-Hill.

Koontz, H., Weihrich, H. y Cannice, M. (2012). Administración: Una perspectiva global $\mathrm{y}$ empresarial. (14a. ed.). México: McGraw-Hill. https://frh.cvg.utn.edu.ar/pluginfile.php/22766/m od resource/content/1/Administracion una pers pectiva_global_y_empresarial_Koontz.pdf

Landay, J. (2019). Smart interfaces for humancentered AI. Stanford University HumanCentered Artificial Intelligence. Stanford, CA: Stanford University. https://hai.stanford.edu/news/smart-interfaceshuman-centered-ai

Leonardi, P. M., y Vaast, E. (2017). Social media and their affor dances for organizing: A review and agenda for research. Academy of Management Annals, 11(1), 150-188. https://papers.ssrn.com/sol3/papers.cfm?abstract id $=2993824$

Moliere. (1997). El Misántropo. Buenos Aires: Losada.

Morales Muñoz, K. y Abal Medina, P. M. (2020). Precarización de plataformas: El caso de los repartidores a domicilio en España. Psicoperspectivas, $19(1)$, 1-12. https://www.proquest.com/docview/2436133793 /5FFAE153C87245DBPQ/1?accountid=31491

Pasquale, F. (2015). The algorithmic self. The Hedgehog Review, 17(1), 30-46. https://digitalcommons.law.umaryland.edu/fac_p ubs/1540/

Perelman, L. (2020). El futuro del trabajo ya llegó: ¿qué hacemos con él? Nueva Sociedad. https://nuso.org/articulo/trabajadores-deplataformas-entre-la-pandemia-y-los-derechos/

Rosenblat, A. y Stark, L. (2016). Algorithmic labor and information asymmetries: A case study of Uber's drivers. International Journal of Communication, $\quad 10, \quad 3758-3784$. https://ijoc.org/index.php/ijoc/article/view/4892/ 1739

Sastre, P. y Gordo López, A. (2019). El activismo de datos frente al control algorítmico. Nuevos modelos de gobernanza, viejas asimetrías. Revista Científica de Información y Comunicación, 16, 157-182. https://icjournal-ojs.org/index.php/ICJournal/article/view/497 
Stoner, J. Freeman, R. y Gilbert, D. (1996). Administración. (6a. ed.). México: Prentice Hall.

Uber Technologies, Inc. (2021, 30 de noviembre). Un mundo de oportunidades. Uber Technologies, Inc. (https://www.uber.com/co/es/drive/

Ulises Mejías, N. C. (2019). Colonialismo de datos: repensando la relación de los datos masivos con el sujeto contemporáneo. Virtualis, Revista de Cultura Digital, 10(18), 78-97. https://www.revistavirtualis.mx/index.php/virtua lis/article/view/289

Wood, A y Lehdonvirta, V. (2021, 31 de marzo). Platform Precarity: surviving algorithmic insecurity in the gig economy. SSRN. https://ssrn.com/abstract $=3795375$ or http://dx.doi.org/10.2139/ssrn.3795375

Woodcock, J. (2020). The algorithmic panopticon at Deliveroo: Measurement, precarity, and the illusion of control. Ephemera. Theory \& Politics in Organization, 20(3), 67-95. www.ephemerajournal.org/contribution/algorith mic-panopticon-deliveroo-measurementprecarity-and-illusion-control-0 